$\checkmark$ Research Square

\title{
Road traffic density and recurrent asthma emergency department visits among Medicaid enrollees in New York State 2005-2015
}

Tabassum Z Insaf ( $\nabla$ tabassum.insaf@health.ny.gov )

New York State Department of Health https://orcid.org/0000-0003-4725-2515

\section{Temilayo Adeyeye}

New York State Department of Health

Catherine Wargo

New York State Department of Health

Victoria Wagner

New York State Department of Health

Anisa Proj

New York State Department of Health

Susan McCauley

New York State Department of Health

Jacqueline Matson

New York State Department of Health

\section{Research Article}

Keywords: Disparities, Asthma, traffic, socioeconomic, race/ethnicity

Posted Date: January 28th, 2022

DOI: https://doi.org/10.21203/rs.3.rs-1261825/v1

License: (9) This work is licensed under a Creative Commons Attribution 4.0 International License.

Read Full License 


\section{Abstract \\ Background}

Environmental exposures such as traffic may contribute to asthma morbidity including recurrent emergency department (ED) visits. However, these associations are often confounded by socioeconomic status and health care access.

\section{Objective}

This study aims to assess the association between traffic density and recurrence of asthma ED visits in the New York State (NYS) Medicaid population between 2005 and 2015.

\section{Methods}

The primary outcome of interest was a recurrent asthma ED visit within 1-year of index visit. Traffic densities (weighted for truck traffic) were spatially linked based on home addresses. Bivariate and multivariate logistic regression analyses were conducted to identify factors predicting recurrent asthma ED visits.

\section{Results}

In a multivariate model, Medicaid recipients living within 300-meters of a high traffic density area were at a statistically significant risk of a recurrent asthma ED visit compared to those in a low traffic density area (OR=1.31; $95 \% \mathrm{Cl}: 1.24$ - 1.38). Additionally, being male (OR=1.24; 95\% Cl:1.20 - 1.27), being on cash assistance $(\mathrm{OR}=1.27 ; 95 \% \mathrm{Cl}: 1.24-1.31)$ and receiving supplemental security income $(\mathrm{OR}=1.23$; $95 \% \mathrm{Cl}: 1.18$ - 1.27) was associated with a higher risk of recurrent asthma ED visit. Black non-Hispanics $(\mathrm{OR}=1.20 ; 95 \% \mathrm{Cl}: 1.10-1.31)$, Hispanics (OR=1.52; 95\% Cl:1.46 - 1.59) and those with race listed as "Other" (OR=1.58; $95 \% \mathrm{Cl}: 1.48$ - 1.68) had higher risk of recurrent asthma ED visits as compared to White non-Hispanics.

\section{Conclusion}

We observed significant persistent disparities in asthma morbidity related to traffic exposure and race/ethnicity in a low-income population. Our findings suggest that even within a primarily low-income study population, socioeconomic differences persist. These differences in susceptibility in the extremely low-income group may not be apparent in health studies that use being on Medicaid as a proxy for low income 


\section{Introduction}

Asthma exacerbations are considered measures of asthma control and quality of care [1, 2]. When exacerbations result in multiple emergency department (ED) visits, they burden the healthcare system and negatively impact patients' quality of life [1]. Efforts to reduce such recurrent visits through coordination of services and controlling exposure to indoor and outdoor environmental triggers can decrease health care costs [3]. Asthma morbidity has been linked with poor ambient air quality. Previous studies have found that residence in urban/inner city areas is associated with increased asthma morbidity such as recurrent ED visits even if it may not be associated with a higher baseline prevalence of asthma $[4,5]$.

As with any environmental or ecological exposure, the measurement of air pollution remains a challenge. Associations of asthma with air pollution are often derived from ecological studies which generalize data from single monitors across wide areas to represent exposure values for individuals living in those areas [6]. The use of individual measures based on residential location can provide a refinement of exposure estimates. Additionally, local traffic may be an important factor even if the area meets federal air quality standards for ozone and annual fine particulate matter $\left(\mathrm{PM}_{2.5}\right)$ and does not have exceedances of the 24hour $\mathrm{PM}_{2.5}$ standard as measured by air monitors [7]. Despite declining regional levels of pollution as measured by central monitors, increase in traffic exposures has led to increase in contribution of traffic as a source of air pollution and an increase in the population exposed to such pollution beyond the metropolitan areas [8]. Key components of emissions from traffic sources such as fine particulate matter ( $\mathrm{PM}<2.5$ or $\mathrm{PM}_{2.5}$ ), volatile organic compounds (VOCs), nitrogen oxides $\left(\mathrm{NO}_{\mathrm{x}}\right)$, carbon monoxide (CO), sulfur dioxide $\left(\mathrm{SO}_{2}\right)$, and ammonia $\left(\mathrm{NH}_{3}\right)$, and other ultrafine and nanoparticles, along with other stressors such as noise, may contribute to multiple health outcomes including asthma [9-13]. Measures of proximity to traffic, specifically those accounting for traffic density and truck traffic, may therefore be a more accurate measure of exposure to Traffic-Related Air Pollution (TRAP) $[7,14,15]$.

In the United States, the poor and those belonging to racial-ethnic minorities are more likely to live very close to major highways with increased traffic flow and thus face a high burden of traffic exposure. Trucks and other large vehicles are often routed through these communities and may contribute to a higher burden of traffic. Socio-economic status (SES) characteristics have been found to be associated with increased susceptibility to asthma. Medicaid enrollment is often used as a proxy for SES in the United States. However, few studies have explored the added burden of race/ethnicity, access to care, and traffic exposures within this population.

This study used Medicaid claim and encounter data and geospatial tools to determine TRAP exposure patterns. We assessed the association between traffic density and the recurrence of ED visits in the New York State (NYS) Medicaid population between 2005 and 2015.

\section{Methods}

\section{Study Population}


NYS Medicaid encounter data, submitted by Medicaid managed care (MMC) health plans, and postadjudicated fee-for-service (FFS) claim data were used to identify Medicaid enrollees less than 65 years of age who had ED visits with a primary diagnosis of asthma (ICD-9 code 493.xx, ICD-10 code J45; CPT Codes: 99221-99223, 99231-99233, 99238, 99239, 99251-99255, 99261-99263, 99291) between 2005 and 2015. Medicaid enrollees with a chronic obstructive asthma diagnosis (ICD-9 code 493.2x, ICD-10 code J44.0, J44.1 and J44.9) were excluded $(17,488)$. Most $(99.04 \%)$ enrollee home addresses at the time of the ED visit were successfully geocoded. Our final study sample consisted of 713,245 ED visits among 296,618 unique Medicaid recipients.

\section{Outcome}

The primary outcome of interest was a recurrent asthma ED visit within 1 year of the index visit. The number of ED visits within a year was calculated for each patient to create the dichotomous outcome variable. Any patient with two or more asthma ED visits in a year was assigned a value of 1 ; patients with a single asthma ED visit in a year were assigned a value of 0 .

Demographic variables included: age group ( $\leq 17,18-44$ and $45-64)$; gender; race/ethnicity (White nonHispanic, Black non-Hispanic, Asian, Native American, Hispanic, and Other); Medicaid coverage program (MMC or FFS); cash assistance (financial assistance provided by NYS); and Supplemental Security Income (SSI) (federal financial assistance for the aged, blind, or disabled). Cash assistance serves as a socioeconomic status (SES) proxy within the Medicaid population.

\section{Traffic Exposure}

The revised 2016 data of Annual Average Daily Traffic (AADT) from the New York State Department of Transportation were used to create multiple measures of traffic: UWTD (unweighted traffic density), TTD (truck traffic density), and WTD (weighted traffic density). UWTD was an unweighted measure taken directly from the 2016 AADT data and includes the total number of vehicles on a segment of road each day. The TTD is an estimate of truck exposure determined from percentage of trucks for that road segment. Trucks were defined as vehicles belonging to Vehicle Classification Codes F04 (Buses) through F13 (Seven-or-More Axle, Multi-Trailer Trucks)[16]. Finally, the WTD was a weighted estimate calculated by including a multiplier for trucks on a road segment to account for the higher emissions associated with truck traffic. Traffic density was then calculated for each traffic count using the Line Density function within ArcMap (ArcGIS Desktop, Version 10.5, Esri, Redlands, CA) to generate a raster with resolution of 50 meters and search radii of 300 meters to produce datasets where the value of each raster cell reflected all traffic on the roads within $300 \mathrm{~m}$ from the centroid. Thus, the impact of any single roadway on a cell's traffic density depends on the amount of traffic on that road segment and its distance from the center of the raster cell. The unit for traffic density is the number of vehicles per square meter per day.

Traffic densities and health outcomes data were spatially linked to assign traffic exposure at the individual level based on home addresses. Unique geographic coordinates of the addresses were mapped as points, and traffic density values for the grid within which each point was enclosed were assigned to 
that point. All addresses outside the specified search radius received a traffic density value of zero (0) and were considered unexposed to vehicle-related emissions at that search distance.

To explore the relationship between different levels of traffic density and recurrent asthma ED visits, tertiles were determined from the statewide distributions of the three traffic density variables and assigned labels of "Low," "Medium," and "High" exposure; those individuals with a traffic density value of zero (0) were incorporated into the "Low" exposure referential group. An inverse hyperbolic sine transformation was applied to traffic density counts to evaluate the association between recurrent asthma visits and traffic density as a continuous variable, maintaining no exposure as a legitimate level of exposure to traffic density. The inverse hyperbolic sine transformation can be used to transform heavily right-skewed data containing negative or zero values, approximating a log-transformation for values strictly greater than zero (0) [17]. As the values of traffic density are strictly non-negative, the results of regressions are interpreted as the probability of experiencing recurrent asthma ED visits with a ten percent increase in traffic density.

\section{Statistical Analysis}

The association between recurrent asthma ED visits and traffic density was calculated using median and interquartile range (IQR) since the data were not normally distributed. We calculated the baseline characteristics of the study population to estimate the median and IQR for continuous variables, or percentages of categorical variables in total and by the outcome variable.

Bivariate and multivariate logistic regression analyses were conducted to identify significant factors that affect recurrent asthma ED visits. To evaluate the robustness of the findings, we performed the multivariate regression analysis with each traffic measure separately. The results were represented as the odds ratio (OR) and $95 \%$ confidence intervals $(\mathrm{Cl})$. Statistical analyses were performed using SAS version 9.4. Confounding was assessed if a $10 \%$ change in estimate was observed when a covariate was removed from the model. Effect modification was estimated by adding interaction terms with a traffic measure for selected covariates. All statistical tests were two-sided, and $p$ values $<0.05$ were considered statistically significant.

\section{Results}

Out of 296,618 Medicaid enrollees with asthma ED visits during the study period, $16.58 \%$ had at least one recurrent asthma ED visit within a year. The study cohort was more likely to be female (58.12\%), between the ages of $18-44$ (42.16\%), Black non-Hispanic (29.99\%), and be enrolled in MMC (86.07\%) (Table 1). The study cohort also lived in areas with a traffic density higher than the state average (Table 2).

Approximately $91 \%$ of the ED visits in NYS (combined) occurred among Medicaid recipients living within 300 meters of a high WTD area during the study period (Table 3). Since New York City (NYC) is densely populated and therefore its inclusion heavily skews the distribution, we also estimated risks for NYS excluding NYC. When NYC is excluded, about $74 \%$ of the ED visits occurred in recipients living within 300 
meters of a high WTD area, $12 \%$ in medium WTD areas, and 15\% in low WTD areas. Approximately $54 \%$ of the ED visits in NYS occurred among recipients living in a high TTD area. However, when NYC was excluded, only $11 \%$ of ED visits occurred among those in a high TTD area.

In the unadjusted analysis, Medicaid recipients who lived within 300 meters of a high traffic density area had a statistically significant higher risk for a recurrent asthma ED visit compared to those who lived in a low WTD area for all three models (for WTD model OR=1.60 95\% Cl:1.53-1.67) (Table 4).

After adjusting for potential confounders (sex, age, race/ethnicity, cash assistance, SSI, and managed care), we observed significantly higher risk for a recurrent asthma ED visit when recipients lived in a high traffic density area in all three models. The risk was higher for medium traffic density areas for truck traffic only in the adjusted models (Table 4). The results were less robust for adjusted models accounting for truck traffic when NYC was excluded.

In both the unadjusted and adjusted models (UWTD and WTD) where Inverse Hyperbolic Sine Transformation was carried out (Table 5), we observed significant risk of recurrent asthma ED visits in both medium and high traffic density areas for NYS (combined) and NYS excluding NYC. In the TTD assessment for NYS (combined), for every $10 \%$ change in traffic density, the risk of recurrent asthma ED visits ranged from $7-16 \%$ and $0-11 \%$ for the unadjusted and adjusted models, respectively (Table 5). We ran unadjusted and adjusted models for all three measures of traffic density with similar results in terms of covariates. We therefore only present full results for WTD models (Table 6). In a multivariate model adjusted for traffic, being male was associated with higher risk of recurrent asthma ED visits. Being of younger or older age had a lower risk of a recurrent asthma ED visit than being in the 18-44 age group. As compared to White non- Hispanics, Black non-Hispanics had the highest risk of recurrent asthma ED visits followed by Hispanics and those of "Other" race/ethnicity categories. Those on cash assistance and recipients of SSI had a higher risk of recurrent asthma ED visits than those who did not receive aid. Those insured by Medicaid FFS were at a higher risk of recurrent asthma ED visits, however the association lost significance after multivariate adjustment.

Finally, we assessed effect modification of the risk associated with exposure to traffic density by other covariates (Figure 2). In low traffic areas, males were at a higher risk of recurrent asthma ED visits but when exposed to medium or high traffic density, females were at a higher risk. Those receiving cash assistance were at a higher risk of recurrent asthma ED visits (Table 6). However, those who did not receive cash assistance were more likely to experience a recurrent asthma ED visit in medium and high traffic density areas. Finally, in low traffic areas children less than 17 years old were at the lowest risk of a recurrent asthma ED visit while in high traffic areas adults between 18-44 years old had the highest risk of recurrent visits.

\section{Discussion}

In a large study of almost 300,000 low-income Medicaid enrollees with asthma in New York State from 2005-2015, we found that those living in high-density traffic areas were at higher risk of recurrent asthma 
ED visits compared to those residing in low-density traffic areas. We found that in the WTD model, exposure to high traffic density areas conferred a 31\% higher risk of recurrent asthma ED visits than those in a low traffic density area even after adjustment for socio-demographic factors. We also found that racial/ethnic disparities in asthma morbidity persist within this low-income population after adjustment for traffic and other contributing factors. We additionally found evidence of effect modification on the risk due to traffic exposure by certain demographic characteristics. Our study therefore suggests that the interplay of environmental and sociodemographic risk factors may result in a much higher risk of asthma morbidity among specific segments of the most vulnerable. Our study found that exposure to both medium and high traffic density may result in an increased risk of recurrent asthma ED visits. Truck traffic also appears to confer an additional risk as evidenced by the higher risk in the WTD and TTD models. It is likely that the increase in asthma morbidity among high traffic areas may also reflect other environmental conditions in urban inner-city neighborhoods, such as exposure to second-hand smoke, poor building conditions, and proximity to toxic release facilities.

TRAP has been suggested to cause physiological damage that can trigger asthma. First, inhaled PM can cause oxidative stress in macrophage and epithelial cells, resulting in airway structural damage. Ultrafine $\mathrm{PM}<100 \mathrm{~nm}$ in diameter can directly damage mitochondria structurally, impair ATP production, and induce apoptosis in macrophages and epithelial cells. Third, PM has the potential to trigger an immunological cascade response to oxidative stress when antioxidant responses are overwhelmed. These pathways can lead to an adverse respiratory event in individuals with asthma, particularly given evidence for reduced antioxidant enzyme capacity in the lungs and peripheral blood of patients with asthma.[18]

Environmental studies have also found that particle concentrations from traffic-related air pollutants (ultrafine PM, black carbon, and CO) generally dissipate by half at around 150 meters and decline to background concentrations by 300 meters from the upwind source road during the day [19]. This means that individuals who live closest to roads with high traffic density are exposed to the most traffic-related air pollution. However, we did find that medium traffic density areas conferred a high risk as well. We also tested measures between 150-300 meters and found similar results.

Most studies of traffic-related pollution focus on a specific urban area. In our study, the most urban area was NYC with $70 \%$ of its population residing in high traffic density regions. We found the highest effect estimates and highest precision for high truck TD areas in NYC after adjusting for confounders (results not presented). Furthermore, we used traffic density measures across the large and geographically diverse state of New York. We were therefore able to look at risk associated with TRAP in the densely populated areas of metropolitan NYC, as well as smaller urban and rural areas across the rest of the state. Our results confirm that TD contributes to significant health risk even in areas with much lower traffic density than large population centers such as NYC.

We also found that those in the 18-44 age group were at a significantly higher risk of recurrent asthma ED visits at baseline levels and were at the highest risk when exposed to high traffic. This may reflect 
increased risk due to direct occupational exposures to high traffic density compared to those who are younger or older living in similar areas. We found that racial/ethnic disparities in asthma persisted in this low-income population even after adjustment for traffic and other sociodemographic factors. These disparities are likely of a complex origin and cannot be attributed to a single cause. For example, because of the historical systemic discrimination and segregation including redlining, vulnerable racial/ethnic minorities may have lower assets, poorer housing conditions, and poorer health status compared to White non-Hispanics of a similar socio-economic status. However, we did not find any significant interactions between traffic and race/ethnicity.

Although males were more likely to have recurrent asthma ED visits in the multivariate adjusted model, we found that females were at a much higher risk of recurrent asthma ED visits when they resided in medium and high traffic density areas as compared to males residing in similar areas. A recent study in California also found that the association between the risk of multiple hospital encounters and living near areas of heavy traffic was higher among female children, although that interaction was not statistically significant [20].

In New York State, Medicaid members with very low incomes such as due to homelessness or disability are eligible for cash assistance. We found that those who received cash assistance were more likely to have asthma exacerbations than those who did not and traffic exposures did not confer an added risk of asthma exacerbation. However, among those who did not receive cash assistance individuals in high traffic density areas were at higher risk of experiencing a recurrent asthma ED visit compared to those exposed to low traffic density (Figure 2). Those who do not qualify for cash assistance may be more likely to engage in occupations or activities that may lead to direct exposure to traffic and therefore confer a higher risk of recurrent asthma ED visits.

The study results agree with the SES-asthma association suggested by previous studies [21]. Our findings suggest that even within this primarily low-income study population socioeconomic differences persist. These differences in susceptibility in the extremely low-income group may not be apparent in health studies that use being on Medicaid as a proxy for low income. Low SES is linked to various health outcomes, with higher rates of morbidity and mortality in chronic diseases such as cardiovascular disease [22], diabetes [23] and chronic obstructive pulmonary disease [24]. In addition, SES may be particularly relevant to asthma in the various pathways through which it may adversely impact asthma health outcomes. Individuals with asthma and lower SES may have higher exposures to both indoor (e.g. mold, cockroaches and tobacco) and outdoor triggers such as pollution [21]. This in turn may increase the risk of asthma exacerbations in such individuals.

Results from this study could be leveraged by Medicaid programs in all 50 states to help identify members with asthma who are most susceptible to the harmful effects of traffic pollution. Understanding the associated risk among minorities and the complex relationship between level of exposure and member characteristics such as sex and receipt of cash assistance, could help Medicaid programs and 
managed care plans alike facilitate the targeted provision of case management and asthma selfmanagement educational services.

There are some limitations to keep in mind when interpreting the findings of our study. Our measure of traffic-related air pollution was ecological. We did not have individual measures of exposure, which may lead to misclassification of exposure. We did not have information on other environmental triggers. Datasets on housing conditions or proximity to local sources of non-traffic air pollution in New York only have limited availability and may not be comprehensive. However environmental triggers may also be more prevalent in poorer neighborhoods which would also be likely to have high traffic exposure [25]. Thus, our effect estimates may also reflect the added burden from these environmental exposures in the most vulnerable populations. The Medicaid claims and encounter data used in this study included diagnostic and procedure data. While underreporting is possible, its effects are likely small as Medicaid reimbursement is determined, in part, by member acuity of illness, which is driven by reported diagnoses and procedures. As has been reported by others, race/ethnicity reporting in Medicaid data can be unreliable $[4,26]$. Eighteen percent of our study subjects had missing race/ethnicity information. This percentage increased toward the end of the study period when more individuals were applying for Medicaid through New York's online health exchange which collects race and ethnicity as optional fields. However, the proportion of individuals with missing race/ethnicity did not differ from those without missing information in terms of traffic exposures. However, those with missing race/ethnicity data were more likely to be $<17$ years of age, and not receiving cash assistance or SSI (Appendix A1). Since those with missing race/ethnicity are likely to be Blacks or Hispanics [27], exclusion of those with missing data would have likely skewed our results towards the null and the underlying associations are probably stronger than reported here.

\section{Conclusion}

Our comprehensive study of asthma morbidity in Medicaid enrollees in New York State found that exposure to high traffic density is associated with increased risk of asthma ED visits. The risk may be even higher in specific subgroups due to physiological susceptibility to higher risk of exposure. Racial and sociodemographic disparities persist in this low-income population even after adjustment for traffic exposures. Our findings suggest that despite significant attention given to asthma in inner city neighborhoods, environmental risk factors such as local traffic can have a higher risk among the most vulnerable. Understanding where this risk is greatest can facilitate the targeted investment of resources to improve patient education and self-management. However, it is only through policies that reduce near road-way pollution exposure including indoor air filtration, relocation of air intakes, and the use of trees and scrubs to serve as vegetative barriers, as well as the re-evaluation of federal emission standards and continued investment in clean diesel programs that the risk will be mitigated. New York State is currently implementing provisions of the Climate Leadership and Community Protection Act, which commits the state to reducing greenhouse gas emissions in a manner that ensures that all New Yorkers, including the most vulnerable, experience the benefits. These findings may be useful in supporting policies which encourage a transition to cleaner transportation options. 


\section{Abbreviations}

AADT

Annual Average Daily Traffic

Cl

Confidence Interval

CO

Carbon Monoxide

ED

Emergency Department

FFS

Fee-For-Service

ICD

International Classification of Diseases

IQR

Interquartile Range

MMC

Medicaid Managed Care

$\mathrm{NH}_{3}$

Ammonia

$\mathrm{NO}_{\mathrm{X}}$

Nitrogen Oxides

NYC

New York City

NYS

New York State

OR

Odds Ratio

$\mathrm{PM}_{2.5}$

Fine Particulate Matter

SES

Socio-economic Status

$\mathrm{SO}_{2}$

Sulfur Dioxide

SSI

Supplemental Security Income

TRAP

Traffic-Related Air Pollution

TTD

Truck Traffic Density 
UWTD

Unweighted Traffic Density

VOCs

Volatile Organic Compounds

WTD

Weighted Traffic Density

\section{Declarations}

\section{Funding:}

This project was supported by the CDC's Environmental Public Health and Emergency Response Program, NYS Unique Federal Award Number NUE1EH001341 (NYS Environmental Public Health Tracking Network Maintenance and Enhancement to Accommodate Sub-County Indicators).

\section{Authors' Contributions:}

TI conceived, designed, and supervised the study and led with manuscript writing. TA conducted the data analysis and co-led the manuscript writing. $\mathrm{CW}$ helped with manuscript writing, data management relating to geographical information systems and mapping. AP also assisted with data extraction and management. VW, SM and JM contributed to the study design. All authors contributed substantial edits to the final version of the article. All authors read and approved the final manuscript.

\section{Corresponding Author}

All correspondence to Tabassum Insaf: Tabassum.insaf@health.ny.gov

\section{Ethical Declarations}

\section{Ethics Approval and Consent to Participate}

The New York State Department of Health (NYSDOH) Institutional Review Board and the New York State Medicaid Data Protection Review Board have approved the access of individually identifiable information such as address, date of birth, and date of hospital visit for use in this study.

\section{Consent for Publication:}

Not applicable

\section{Competing Interests:}

The authors declare no competing interests.

\section{Availability of data and materials:}


The health data that support the findings of this study are available from the Office of Health Insurance Programs (OHIP), New York State Department of Health (NYSDOH), but restrictions apply to the availability of these data, which were used under license for the current study, and so are not publicly available. Data are however available from the authors upon reasonable request and with permission of OHIP, NYSDOH.

The Annual Average Daily Traffic (AADT) data is available from the New York State Department of Transportation Traffic Data Viewer at https://www.dot.ny.gov/tdv

\section{References}

1. Suruki RY, Daugherty JB, Boudiaf N, Albers FC: The frequency of asthma exacerbations and healthcare utilization in patients with asthma from the UK and USA. BMC Pulmonary Medicine 2017, 17(1):74.

2. Baltrus P, Xu J, Immergluck L, Gaglioti A, Adesokan A, Rust G: Individual and county level predictors of asthma related emergency department visits among children on Medicaid: A multilevel approach. J Asthma 2017, 54(1):53-61.

3. Kercsmar CM, Beck AF, Sauers-Ford H, Simmons J, Wiener B, Crosby L, Wade-Murphy S, Schoettker PJ, Chundi PK, Samaan Z et al: Association of an Asthma Improvement Collaborative With Health Care Utilization in Medicaid-Insured Pediatric Patients in an Urban Community. JAMA Pediatrics 2017, 171(11):1072-1080.

4. Keet CA, Matsui EC, McCormack MC, Peng RD: Urban residence, neighborhood poverty, race/ethnicity, and asthma morbidity among children on Medicaid. Journal of Allergy and Clinical Immunology 2017, 140(3):822-827.

5. Keet CA, McCormack MC, Pollack CE, Peng RD, McGowan E, Matsui EC: Neighborhood poverty, urban residence, race/ethnicity, and asthma: rethinking the inner-city asthma epidemic. Journal of Allergy and Clinical Immunology 2015, 135(3):655-662.

6. Szyszkowicz M, Kousha T, Castner J, Dales R: Air pollution and emergency department visits for respiratory diseases: A multi-city case crossover study. Environmental Research 2018, 163:263-269.

7. Kim Janice J, Huen K, Adams S, Smorodinsky S, Hoats A, Malig B, Lipsett M, Ostro B: Residential Traffic and Children's Respiratory Health. Environmental Health Perspectives 2008, 116(9):12741279.

8. Buzzelli M, Jerrett M, Burnett R, Finklestein N: Spatiotemporal perspectives on air pollution and environmental justice in Hamilton, Canada, 1985-1996. Annals of the Association of American Geographers 2003, 93(3):557-573.

9. Campbell P, Zhang Y, Yan F, Lu Z, Streets D: Impacts of transportation sector emissions on future U.S. air quality in a changing climate. Part l: Projected emissions, simulation design, and model evaluation. Environmental pollution (Barking, Essex : 1987) 2018, 238:903-917. 
10. Plato N, Bigert C, Larsson BM, Alderling M, Svartengren M, Gustavsson P: Exposure to Particles and Nitrogen Dioxide Among Workers in the Stockholm Underground Train System. Safety and health at work 2019, 10(3):377-383.

11. Bowatte G, Lodge CJ, Knibbs LD, Lowe AJ, Erbas B, Dennekamp M, Marks GB, Giles G, Morrison S, Thompson $B$ et al: Traffic-related air pollution exposure is associated with allergic sensitization, asthma, and poor lung function in middle age. Journal of Allergy and Clinical Immunology 2017, 139(1):122-129.e121.

12. Bowatte G, Lodge CJ, Knibbs LD, Erbas B, Perret JL, Jalaludin B, Morgan GG, Bui DS, Giles GG, Hamilton GS et al: Traffic related air pollution and development and persistence of asthma and low lung function. Environment International 2018, 113:170-176.

13. Lee YJ, Rabinovitch N: Relationship between traffic-related air pollution particle exposure and asthma exacerbations: Association or causation? Annals of Allergy, Asthma \& Immunology 2018, 120(5):458-460.

14. Ferguson EC, Maheswaran R, Daly M: Road-traffic pollution and asthma - using modelled exposure assessment for routine public health surveillance. International journal of health geographics 2004, $3(1): 24-24$.

15. Mosnaim G, Wi C-I, Wheeler PH, Ryu E, King KS, Park MA, Juhn YJ: Geospatial Analysis for Assessing the Impact of High Traffic Volume on Asthma Exacerbations in a Mixed Rural-Urban US Community. Journal of Allergy and Clinical Immunology 2019, 143(2):AB210.

16. Vehicle Classification Codes [https://www.dot.ny.gov/divisions/engineering/applications/trafficdata-viewer/tdv-definitions/Vehicle-Classification-Codes.htm]

17. Zhang M, Fortney JC, Tilford JM, Rost KM: An Application of the Inverse Hyperbolic Sine Transformation-A Note. Health Services and Outcomes Research Methodology 2000, 1(2):165-171.

18. Miller MR, Raftis JB: Evidence from toxicological and mechanistic studies. In: Traffic-Related Air Pollution. Elsevier; 2020: 229-279.

19. Zhu Y, Hinds WC, Kim S, Sioutas C: Concentration and size distribution of ultrafine particles near a major highway. Journal of the air \& waste management association 2002, 52(9):1032-1042.

20. Delfino RJ, Chang J, Wu J, Ren C, Tjoa T, Nickerson B, Cooper D, Gillen DL: Repeated hospital encounters for asthma in children and exposure to traffic-related air pollution near the home. Annals of Allergy, Asthma \& Immunology 2009, 102(2):138-144.

21. Bacon SL, Bouchard A, Loucks EB, Lavoie KL: Individual-level socioeconomic status is associated with worse asthma morbidity in patients with asthma. Respiratory research 2009, 10(1):125.

22. Cooper R, Cutler J, Desvigne-Nickens P, Fortmann SP, Friedman L, Havlik R, Hogelin G, Marler J, McGovern P, Morosco G et al: Trends and disparities in coronary heart disease, stroke, and other cardiovascular diseases in the United States: findings of the national conference on cardiovascular disease prevention. Circulation 2000, 102(25):3137-3147.

23. Brown AF, Ettner SL, Piette J, Weinberger M, Gregg E, Shapiro MF, Karter AJ, Safford M, Waitzfelder B, Prata PA et al: Socioeconomic Position and Health among Persons with Diabetes Mellitus: A 
Conceptual Framework and Review of the Literature. Epidemiologic Reviews 2004, 26(1):63-77.

24. O'Malley AS, Pham HH, Schrag D, Wu B, Bach PB: Potentially Avoidable Hospitalizations for COPD and Pneumonia: The Role of Physician and Practice Characteristics. Medical Care 2007, 45(6):562570.

25. Wilson SM, Fraser-Rahim H, Williams E, Zhang H, Rice L, Svendsen E, Abara W: Assessment of the distribution of toxic release inventory facilities in metropolitan Charleston: an environmental justice case study. American journal of public health 2012, 102(10):1974-1980.

26. Race and Ethnicity: DQ Assessments [https://www.medicaid.gov/dqatlas/landing/topics/single/map?topic=g3m16]

27. Fernandez LE, Rastogi S, Ennis SR, Noon JM: Evaluating race and Hispanic origin responses of Medicaid participants using census data. Statistical Journal of the IAOS 2018, 34:521-528.

\section{Tables}

Table 1. Frequency Distribution of New York State Medicaid Asthma-Related Emergency Department Patients (2005 - 2015) 


\begin{tabular}{|c|c|}
\hline Variables & $\begin{array}{l}\text { Total } \\
\text { N (\%) }\end{array}$ \\
\hline Asthma Emergency Department Visits & $296,618(100)$ \\
\hline 1 asthma ED visit & $247,425(83.42)$ \\
\hline$\geq 2$ asthma ED visits & $49,193(16.58)$ \\
\hline \multicolumn{2}{|l|}{ Sex } \\
\hline Male & $124,234(41.88)$ \\
\hline Female & $172,384(58.12)$ \\
\hline \multicolumn{2}{|l|}{ Age at first encounter } \\
\hline$\leq 17$ & $118,963(40.11)$ \\
\hline $18-44$ & $125,066(42.16)$ \\
\hline $45-64$ & $52,587(17.73)$ \\
\hline Missing & $2(0.00)$ \\
\hline \multicolumn{2}{|l|}{ Race/Ethnicity } \\
\hline White non-Hispanic & $53,446(18.02)$ \\
\hline Black non-Hispanic & $88,966(29.99)$ \\
\hline Asian & $7,417(2.50)$ \\
\hline Native American & $683(0.23)$ \\
\hline Hispanic & $79,351(26.75)$ \\
\hline Other & $11,635(3.92)$ \\
\hline Missing & $55,120(18.58)$ \\
\hline \multicolumn{2}{|l|}{ Cash Assistance } \\
\hline Yes & $130,731(44.07)$ \\
\hline No & $165,887(55.93)$ \\
\hline \multicolumn{2}{|l|}{ SSI } \\
\hline Yes & $60,353(20.35)$ \\
\hline No & $236,265(79.65)$ \\
\hline \multicolumn{2}{|l|}{ Managed Care } \\
\hline Yes & $255,307(86.07)$ \\
\hline No & $41,311(13.93)$ \\
\hline
\end{tabular}


Table 2. Univariate distribution of traffic density exposure (number of vehicles per square meter per day)

\begin{tabular}{|lllllll|}
\hline & \multicolumn{2}{l}{ Unweighted Traffic Density } & \multicolumn{2}{l|}{ Weighted Traffic Density } & \multicolumn{2}{l|}{ Truck Traffic Density } \\
\hline Measures & $\begin{array}{l}\text { Study } \\
\text { Population }\end{array}$ & $\begin{array}{l}\text { Statewide } \\
\text { Distribution }\end{array}$ & $\begin{array}{l}\text { Study } \\
\text { Population }\end{array}$ & $\begin{array}{l}\text { Statewide } \\
\text { Distribution }\end{array}$ & $\begin{array}{l}\text { Study } \\
\text { Population }\end{array}$ & $\begin{array}{l}\text { Statewide } \\
\text { Distribution }\end{array}$ \\
\hline Mean & 76.00 & 11.87 & 89.62 & 13.91 & 5.74 & 1.56 \\
\hline Median & 49.00 & 3.00 & 57.00 & 3.00 & 3.00 & 1.00 \\
$\begin{array}{l}\text { Standard } \\
\text { Deviation }\end{array}$ & 84.65 & 32.73 & 102.23 & 38.53 & 7.23 & 2.45 \\
\hline
\end{tabular}

Table 3. Number of Asthma-Related Emergency Department Visits (2005 - 2015) in New York State Medicaid Population by traffic density

\begin{tabular}{|llll|}
\hline Model & \multicolumn{3}{l}{ Traffic Density @ 300 meters } \\
\cline { 2 - 4 } & Low & Medium & High \\
& $\mathrm{n}(\%)$ & $\mathrm{n}(\%)$ & $\mathrm{n}(\%)$ \\
\hline Weighted & & & \\
\hline NYS & $32,661(4.58)$ & $29,189(4.09)$ & $651,395(91.33)$ \\
\hline NYS excl. NYC & $27,531(14.52)$ & $22,147(11.68)$ & $139,960(73.80)$ \\
\hline Unweighted & & & \\
\hline NYS & $28,579(4.01)$ & $24,089(3.38)$ & $660,577(92.62)$ \\
\hline NYS excl. NYC & $24,123(12.72)$ & $18,140(9.57)$ & $147,375(77.71)$ \\
\hline Trucks only & & & \\
\hline NYS & $134,503(18.86)$ & $196,154(27.50)$ & $382,588(53.64)$ \\
\hline NYS excl. NYC & $105,955(55.87)$ & $63,247(33.35)$ & $20,436(10.78)$ \\
\hline
\end{tabular}

Table 4. Odds Ratios for the Association between Ranked Traffic Density and Asthma Exacerbations 


\begin{tabular}{|c|c|c|c|c|}
\hline \multirow[t]{2}{*}{ Model } & \multicolumn{2}{|l|}{ Unadjusted } & \multicolumn{2}{|l|}{ Adjusted§ } \\
\hline & $\begin{array}{l}\text { Medium Traffic } \\
\text { Density }\end{array}$ & $\begin{array}{l}\text { High Traffic } \\
\text { Density }\end{array}$ & $\begin{array}{l}\text { Medium Traffic } \\
\text { Density }\end{array}$ & $\begin{array}{l}\text { High Traffic } \\
\text { Density }\end{array}$ \\
\hline \multicolumn{5}{|c|}{ Unweighted ${ }^{¥}$} \\
\hline NYS & $1.05(0.98,1.13)$ & $1.58(1.51,1.66)$ & $1.01(0.94,1.09)$ & $1.29(1.22,1.36)$ \\
\hline $\begin{array}{l}\text { NYS excl. } \\
\text { NYC }\end{array}$ & $0.96(0.89,1.04)$ & $1.26(1.19,1.33)$ & $0.94(0.87,1.02)$ & $1.10(1.03,1.17)$ \\
\hline \multicolumn{5}{|l|}{ Weighted $^{\dagger}$} \\
\hline NYS & $1.08(1.01,1.15)$ & $1.60(1.53,1.67)$ & $1.03(0.96,1.11)$ & $1.31(1.24,1.38)$ \\
\hline $\begin{array}{l}\text { NYS excl. } \\
\text { NYC }\end{array}$ & $1.00(0.93,1.07)$ & $1.27(1.20,1.34)$ & $0.96(0.89,1.04)$ & $1.11(1.05,1.18)$ \\
\hline \multicolumn{5}{|c|}{ Trucks only ${ }^{\ddagger}$} \\
\hline NYS & $1.35(1.31,1.39)$ & $1.51(1.46,1.55)$ & $1.23(1.19,1.27)$ & $1.32(1.28,1.37)$ \\
\hline $\begin{array}{l}\text { NYS excl. } \\
\text { NYC }\end{array}$ & $1.17(1.12,1.22)$ & $1.15(1.08,1.22)$ & $1.11(1.06,1.16)$ & $1.05(0.97,1.12)$ \\
\hline
\end{tabular}

§Adjusted for age (ref = $18-44)$, sex $(r e f=F)$, cash assistance $(r e f=$ No), managed care $(r e f=$ No), SSI $($ ref $=$ Non $)$, race $(r e f=$ White non-Hispanic $)$

$¥$ Unweighted rankings: low $(0-1)$, medium $(2-6)$, high $(7-890)$

tWeighted for trucks rankings: low $(0-2)$, medium $(3-9)$, high $(10-1071)$

$\ddagger$ Trucks only rankings: low $(0-1)$, medium $(2-3)$, high $(4-79)$

Table 5. Odds Ratios for the Association between Traffic Density and Asthma Exacerbations (Inverse Hyperbolic Sine Transformation) 


\begin{tabular}{|lll|}
\hline Model & Log Traffic Density (Unadjusted) & Log Traffic Density (Adjusted) $\S$ \\
\hline Unweighted & & \\
\hline NYS & $1.11(1.11,1.12)$ & $1.07(1.06,1.08)$ \\
\hline NYS excl. NYC & $1.07(1.06,1.09)$ & $1.03(1.02,1.05)$ \\
\hline Weighted & & $1.07(1.06,1.08)$ \\
\hline NYS & $1.11(1.10,1.12)$ & $1.03(1.02,1.05)$ \\
\hline NYS excl. NYC & $1.07(1.06,1.08)$ & $1.09(1.08,1.11)$ \\
\hline Trucks only & & $1.03(1.00,1.07)$ \\
\hline NYS & $1.15(1.14,1.16)$ & $1.10(1.07,1.13)$ \\
\hline NYS excl. NYC & $1.03)$ \\
\hline
\end{tabular}

§Adjusted for age (ref = $18-44)$, sex $(r e f=F)$, cash assistance $($ ref $=$ No), managed care $(r e f=$ No), SSI $($ ref $=$ Non $)$, race $($ ref $=$ White non-Hispanic $)$

Table 6. Multivariate associations between traffic density, covariates, and recurrent asthma ED visits in NYS Medicaid population 2005-2015 


\begin{tabular}{|c|c|c|}
\hline Covariates & Unadjusted & Multivariate Adjusted \\
\hline \multicolumn{3}{|l|}{ Traffic (NYS) } \\
\hline High WTD & $1.60(1.53,1.67)$ & $1.31(1.24,1.38)$ \\
\hline Medium WTD & $1.08(1.01,1.15)$ & $1.03(0.96,1.11)$ \\
\hline Low WTD & Ref & Ref \\
\hline \multicolumn{3}{|l|}{ Sex } \\
\hline Male & $1.15(1.12,1.18)$ & \\
\hline \multirow[t]{2}{*}{ Female } & Ref. & $1.24(1.20,1.27)$ \\
\hline & & Ref. \\
\hline \multicolumn{3}{|l|}{ Age } \\
\hline$<17$ & $0.83(0.80,0.85)$ & $0.76(0.74,0.78)$ \\
\hline $18-44$ & Ref. & Ref. \\
\hline $45-64$ & $1.01(0.97,1.04)$ & $0.86(0.82,0.89)$ \\
\hline \multicolumn{3}{|l|}{ Race/Ethnicity } \\
\hline White non-Hispanic & Ref. & Ref. \\
\hline Black non-Hispanic & $1.81(1.74,1.89)$ & $1.68(1.62,1.76)$ \\
\hline Asian & $1.12(1.02,1.22)$ & $1.20(1.10,1.31)$ \\
\hline Native American & $0.97(0.68,1.39)$ & $1.00(0.70,1.44)$ \\
\hline Hispanic & $1.65(1.58,1.72)$ & $1.52(1.46,1.59)$ \\
\hline Other & $1.68(1.57,1.79)$ & $1.58(1.48,1.68)$ \\
\hline \multicolumn{3}{|l|}{ Cash Assistance } \\
\hline Yes & $1.46(1.42,1.49)$ & $1.27(1.24,1.31)$ \\
\hline No & Ref. & Ref. \\
\hline \multicolumn{3}{|l|}{ SSI } \\
\hline Yes & $1.43(1.39,1.47)$ & $1.23(1.18,1.27)$ \\
\hline No & Ref. & Ref. \\
\hline \multicolumn{3}{|l|}{ Managed Care } \\
\hline Yes & Ref. & Ref. \\
\hline No & $1.05(1.01,1.09)$ & $1.03(0.99,1.08)$ \\
\hline
\end{tabular}


*WTD - weighted traffic density

\section{Figures}

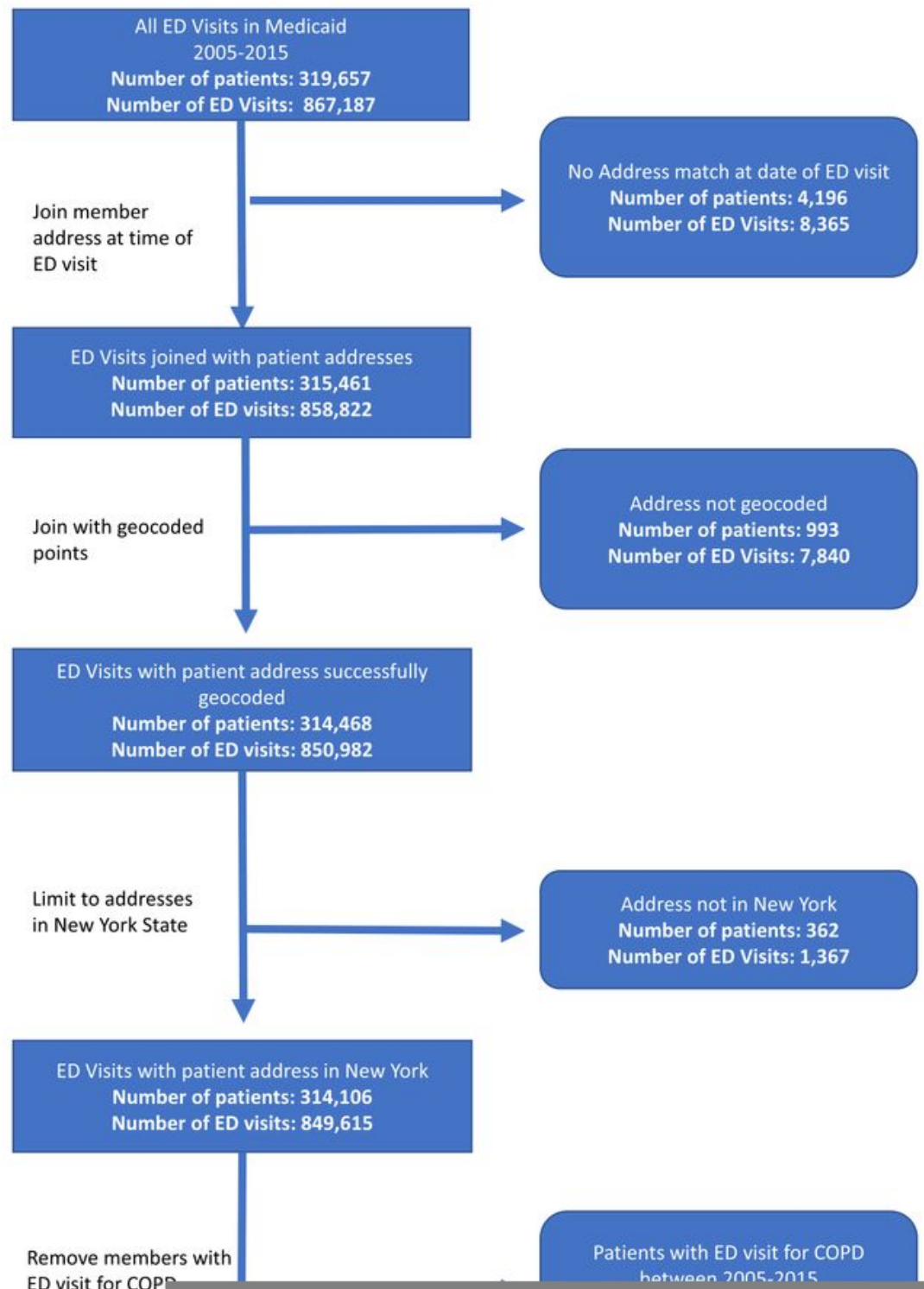

\section{Figure 1}




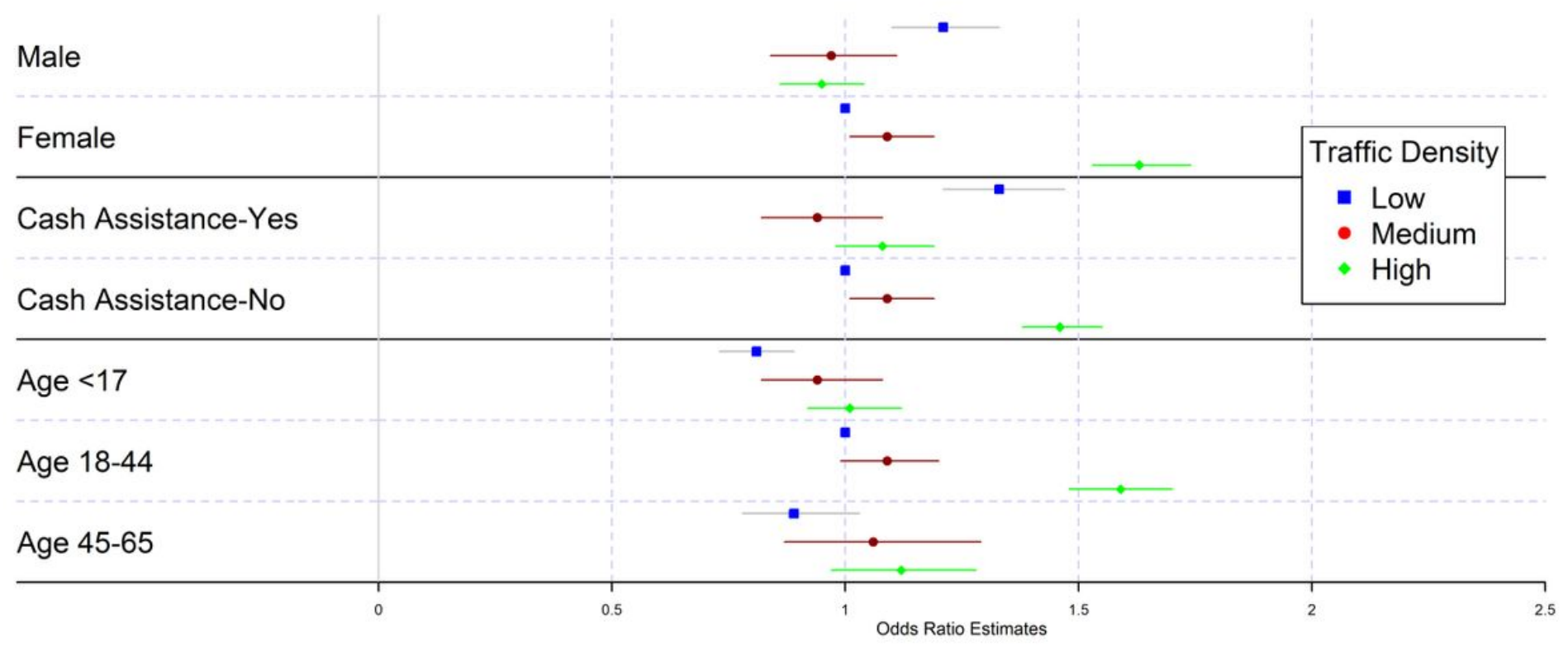

Figure 2

Legend not included with this version

\section{Supplementary Files}

This is a list of supplementary files associated with this preprint. Click to download.

- SupplementaryDocumentAppendix.docx 